\title{
NEW RESULTS ON RESISTANCE-HARARY INDICES
}

\section{Chuantao Li}

School of Science, China University of Geosciences (Beijing), Beijing 100083,

P. R. China

\section{Abstract}

Resistance-Harary index of a connected graph $G$ is defined as

$$
R H(G)=\sum_{\{u, v\} \subseteq V(G)} \frac{1}{r_{G}(u, v)},
$$

where $r_{G}(u, v)$ is the resistance distance between vertices $u$ and $v$ in $G$. It originates from many physical problems. In this paper, we completely determine Resistance-Harary indices of two classes graphs.

Keywords: resistance-Harary index, path, cycle.

\section{Introduction}

Topological indices of molecular graphs are very important invariants of a graph, which can be used to explore properties of a molecular

${ }^{*}$ Corresponding author.

E-mail address: 2012010020@cugb.edu.cn (Chuantao Li).

Copyright @ 2019 Scientific Advances Publishers

2010 Mathematics Subject Classification: 05C05, 05C12.

This research is supported by NSFC (No.11701530).

Submitted by Jianqiang Gao.

Received November 10, 2019 
structured graph. It has been studied by a lot of scientists both in mathematics, physics and in chemistry for many years.

Let $G$ be a simple and connected graph with $|V(G)|=n$ and $|E(G)|=m$. For any $v \in V(G), d(v)$ is the degree of vertex $v$, the distance between vertices $u$ and $v$, denoted by $d(u, v)$, is the length of a shortest path between them. The resistance distance $r(u, v)$ (if more than one graphs are considered, we write $r_{G}(u, v)$ in order to avoid confusion) between vertices $u$ and $v$ in $G$ is defined as the effective resistance between the two nodes of the electronic network obtained so that its nodes correspond the vertices of $G$ and each edge of $G$ is replaced by a resistor of unit resistance, which is computed by the methods of the theory of resistive electrical networks based on Ohm's and Kirchhoff's laws.

In 1993, Klein and Randić [3] defined a new distance function named resistance on the basis of electrical network theory. The Kirchhoff index $K f(G)$ of a graph $G$ is defined as

$$
K f(G)=\sum_{\{u, v\} \subseteq V(G)} r_{G}(u, v)
$$

In 2017, Chen et al. [2] introduced a new graph invariant reciprocal to Kirchhoff index, named Resistance-Harary index, as

$$
R H(G)=\sum_{\{u, v\} \subseteq V(G)} \frac{1}{r_{G}(u, v)},
$$

where $r_{G}(u, v)$ is the resistance distance between vertices $u$ and $v$ in $G$. It originates from many physical problems. Let $P_{n}$ and $C_{n}$ denote Path and Cycle with $n$ vertices, respectively. All other notations and terminologies are referred to [1]. 


\section{Main Results}

In this section, we begin to determine Resistance-Harary indices of Path $P_{n}$ and Star $S_{n}$ with $n$ vertices.

Theorem 1. For any positive integer $n \geq 2, R H\left(P_{n}\right)=n \sum_{i=2}^{n} \frac{1}{i}$.

Proof. Let $V\left(P_{n}\right)=\left\{v_{1}, v_{2}, \cdots, v_{n}\right\}$. According to the structures of Path, any circuit connecting any two vertices is a series circuit. There are in all $(n-1)$ values $1,2, \cdots, n-1$ of $r\left(v_{i}, v_{j}\right)$ in $P_{n}$, and the total numbers are $n-1, n-2, \cdots, 1$, respectively. Then

$$
R H\left(P_{n}\right)=\frac{n-1}{1}+\frac{n-2}{2}+\cdots+\frac{1}{n-1}=n\left(\frac{1}{2}+\cdots+\frac{1}{n}\right)=n \sum_{i=2}^{n} \frac{1}{i} .
$$

Theorem 2. For any positive integer $n \geq 2, R H\left(S_{n}\right)=\frac{(n-1)(n+2)}{4}$.

Proof. Let Star $S_{n}=\left\{v_{1}, v_{2}, \cdots, v_{n}\right\}$, where $v_{1}$ is the center of Star. According to the structures of Star, we have consider two cases as follows:

Case 1. $x, y \in\left\{v_{2}, \cdots, v_{n}\right\} . r(x, y)=2$ and the total number of $r(x, y)$ is $\frac{(n-1)(n-2)}{2}$.

Case 2. $x=v_{1}$ and $y \in\left\{v_{2}, \cdots, v_{n}\right\} . r(x, y)=1$ and the total number of $r(x, y)$ is $(n-1)$. Then

$$
R H\left(S_{n}\right)=\frac{(n-1)(n-2)}{2} \cdot \frac{1}{2}+(n-1) \cdot \frac{1}{1}=\frac{(n-1)(n+2)}{4} .
$$




\section{References}

[1] J. A. Bondy and U. S. R. Murty, Graph Theory with Applications, MacMillan, New York, NY, 1976.

[2] S. Chen, Z. Guo, T. Zeng and L. Yang, On the resistance-Harary index of unicyclic graphs, Communications in Mathematical and in Computer Chemistry 78(1) (2017), 189-198.

[3] D. J. Klein and M. Randić, Resistance distance, Journal of Mathematical Chemistry 12(1) (1993), 81-95.

DOI: https://doi.org/10.1007/BF01164627 